\title{
Brainstem auditory evoked potentials in healthy cats recorded with surface electrodes
}

\author{
Mihai Musteata ${ }^{1}$, Irina Neculae ${ }^{1}$, Mihaela Armasu ${ }^{1}$, Christiana B. Balan², \\ Gheorghe Solcan ${ }^{1}$
}

\begin{abstract}
${ }^{1}$ University of Agricultural Sciences and Veterinary Medicine Ion Ionescu de la Brad Iasi, Faculty of Veterinary Medicine, Department of Internal Medicine / Neurology, Iasi, Romania,

${ }^{2}$ University “A.I. Cuza”, Department of Statistics, Iasi, Romania
\end{abstract}

Received July 7, 2010

Accepted November 29, 2012

\begin{abstract}
The aim of this study was to evaluate the brainstem auditory evoked potentials of seven healthy cats, using surface electrodes. Latencies of waves I, III and V, and intervals I-III, I-V and III-V were recorded. Monaural and binaural stimulation of the cats were done with sounds ranging between 40 and 90 decibel Sound Pressure Level. All latencies were lower than those described in previous studies, where needle electrodes were used. In the case of binaural stimulation, latencies of waves III and V were greater compared to those obtained for monaural stimulation $(P<0.01)$, and relatively unchanged for wave I $(P>0.05)$. Regardless of the sound intensity, the interwave latency was constant $(P>0.05)$. Interestingly, no differences were noticed for latencies of waves III and V when sound intensity was higher than 80dB SPL. This study completes the knowledge in the field of electrophysiology and shows that the brainstem auditory evoked potentials in cats using surface electrodes is a viable method to record the transmission of auditory information. That can be faithfully used in clinical practice, when small changes of latency values may be an objective factor in health status evaluation.
\end{abstract}

BAER, latency, deafness, feline

Evaluation of hearing disorders in cats is of increasing interest, as the prevalence of such disorders is high. An allele of particular interest in this field is the dominant W (white) variant, responsible for white hair in the feline population (at least 14 breeds of cats have a dominant allele of the white gene) (Geigy et al. 2007). The prevalence of deafness in the white coated feline population is high, up to $50 \%$ according to Delack (1984). This allele is also found in cats without pure white coats (Strain 2007).

Brainstem auditory evoked potentials (BAER) are a diagnostic tool used in the evaluation of feline and canine hearing disorders (Wilson and Mills 2005; Besalti et al. 2008; Cvejic et al. 2009). It is often used in conjunction with other diagnostic tests and imaging procedures. Due to the fact that advanced imaging techniques alone are not specific enough to reach a conclusive diagnosis, the BAER test of both peripheral and central structures remains the most useful adjunctive test to assess the physiological integrity of auditory pathways. In veterinary medicine, BAER is represented by four main waves (I, II, III and V) and sometimes a supplementary one (IV). The nuclear generators of the waves are represented by cochlear nerve (wave I), cochlear nucleus and unmyelinated central terminals of the cochlear nerve (wave II), dorsal nucleus of the trapezoid body (wave III), and caudal colliculus (wave V). For wave IV the generators may be placed in the lateral lemniscus (Poma et al. 2008).

However, in the absence of reference values, electrical activity of these structures can only be interpreted by comparison with values obtained contralaterally. This can be problematic in animals with bilateral, and particularly, asymmetrical disease. Different types of electrodes can be used in order to perform a BAER test. Compared to humans

Phone: +40232407456

Fax: +40232219113

E-mail: gsolcan@uaiasi.ro

http://actavet.vfu.cz/ 
where surface electrodes are preferred, in veterinary literature there are many reports on BAER testing with needle electrodes. These different types of electrodes may have an impact in clinical decisions.

To the authors' knowledge, there are no reports of BAER acquired with surface electrodes in cats. Therefore, the aim of this study, is to record the latencies and intervals of BAER at different stimulus intensities using circular surface electrodes in healthy cats.

\section{Materials and Methods}

To evaluate brainstem auditory evoked potentials using surface electrodes, seven cats (both sexes), aged 2-5 years (mean 3.2 years) with no neurological or hearing disorders, were investigated. The use of animals in this study was approved by the Council of Ethics of the University of Agricultural Sciences and Veterinary Medicine Iasi (number 11332/20.07.2009).

The test was made under general anaesthesia with medetomidine hydrochloride (Domitor, Pfizer, Finland) at the dose of $0.05 \mathrm{mg} \cdot \mathrm{kg}^{-1}$ inj. i.m. with Neuropack S, MEB $9400 \mathrm{~K}$ electrodiagnostic system (Nihon Kohden, Japan) in the auditory brainstem response program (ABR). The waves were recorded with surface electrodes placed as follows: the active electrode on the vertex, reference electrodes at the base of each ear and the grounding electrode on the median line, retrooccipitally. The area on which the electrodes were placed was trimmed, degreased with alcohol and Skin Pure NIHON KOHDEN, and covered with special adhesive paste (EEG Paste Elefix ${ }^{\circledR}$ Nihon Kohden).

Impedance was lower than $5 \Omega$. Alternating click stimuli of $0.1 \mathrm{~ms}$ were applied through earphones inserted into the auditory canal. Monoaural and binaural stimulation were performed at different intensities of the stimulus, 90 to $40 \mathrm{~dB}$ SPL (decibel sound pressure level), using steps of $10 \mathrm{~dB}$ SPL (the non-tested ear was masked with white noise of an intensity $40 \mathrm{~dB}$ lower than that used on the tested ear). Each waveform was the average of 500 stimulations, using a High-cut filter of $100 \mathrm{~Hz}$ and a Low-cut filter of $3000 \mathrm{~Hz}$ (Arnold 2007). Artifactual data were automatically rejected; when rejected waveforms represented more than $5 \%$ of the average, the test was repeated. The waves were manually labelled by the same examiner, each positive peak receiving a Roman score from I to V, and latencies of waves I, III, and V were measured, as well as the intervals I-III, III-V and I-V. The test was repeated after 4 days in order to identify any differences. The final value was considered as a mean of those two examinations.

The statistical interpretation of the results was done with the software Statistical Package for the Social Sciences for Windows (SPSS) 16, Wilcoxon Signed Ranks Test for 2 paired samples, with a significance threshold $P<0.05$. The variation coefficient was calculated to verify the homogeneity of the cats included in the study.

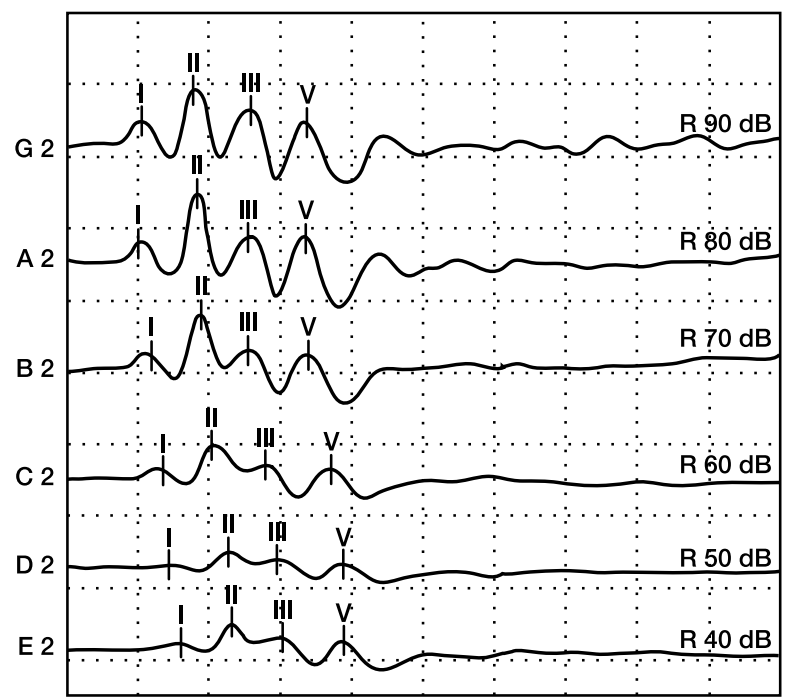

Fig. 1. Typical morphology of brainstem auditory evoked responses recorded after stimulation of the right ear of a cat with a controlled auditive stimulus with intensities between 90 and 40 decibel sound pressure level $(\mathrm{dB})$ 


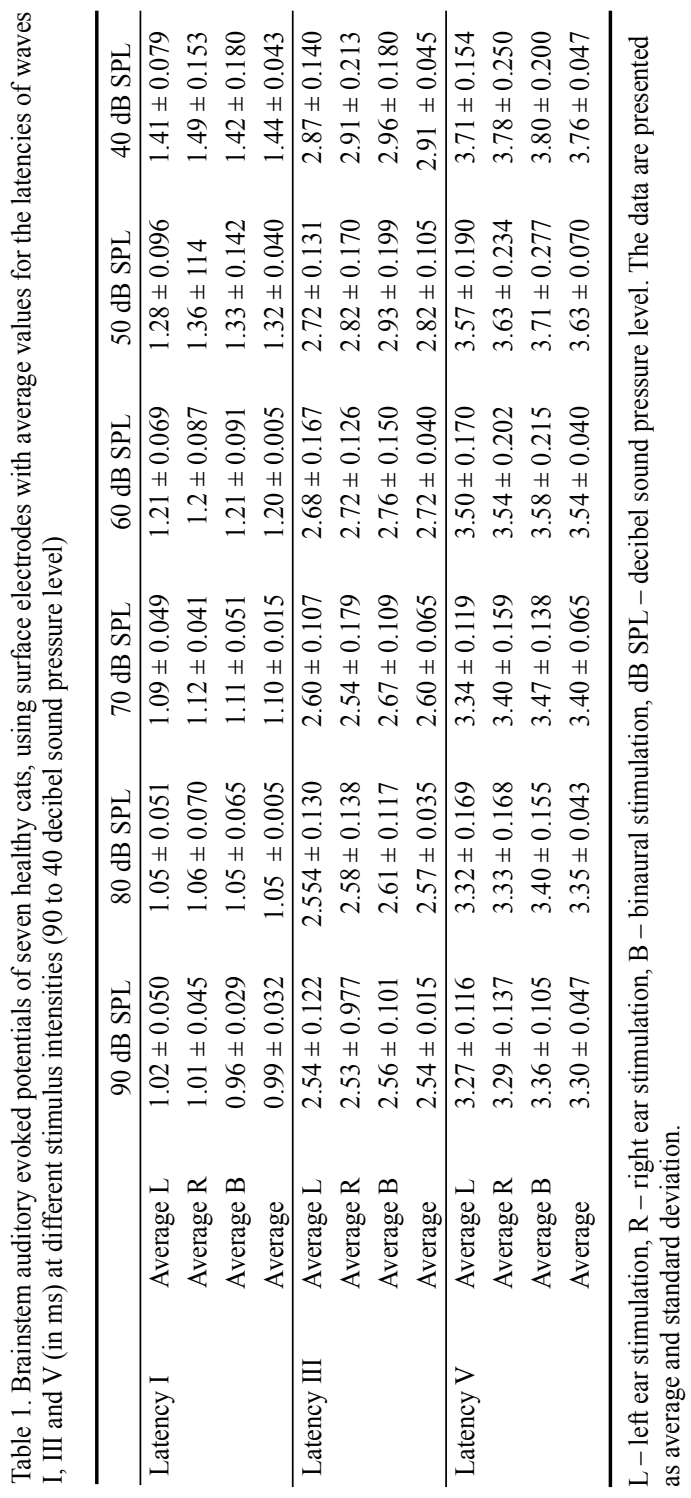

\section{Results}

No significant differences were observed between the two examinations $(P>0.05)$. In all records, BAER waves were characterized by a normal characteristic with I, III and V waves being marked (Fig. 1).

The values of wave latencies and interwave intervals at different sound intensities are presented in Tables 1 and 2.

Comparing the latencies of waves I, III and V generated by left and right monaural stimulation, we did not observe significant differences $(P>0.05)$. However, comparing the latencies of the waves recorded after binaural and monaural stimulation (left or right), we noted significant changes for the latencies of wave III at $80 \mathrm{~dB}$ SPL $(P=0.027)$ and $90 \mathrm{~dB}$ SPL $(P=0.043)$ for the left ear, and $70 \mathrm{~dB}$ SPL $(P=0.043)$ for the right ear. For wave $\mathrm{V}$, differences were observed at $90 \mathrm{~dB}$ SPL $(P<0.05)$ both right and left ear. In all of these situations, the wave latencies after binaural stimulation were longer than for monaural stimulation.

Regarding the latencies of waves I, III and V, we noticed that they have an inverse relation with the intensity of the stimulus, the differences being significant $(P<0.05)$ regardless of the type of stimulation (monaural or binaural), excepting the values obtained for the intensities higher than $80 \mathrm{~dB}$ SPL, when the latency of the waves was not influenced by the increase of the intensity of the sound $(P>0.05)$. In case of the latency of wave $V$ at binaural stimulation, significant changes were not seen at intensities higher than $80 \mathrm{~dB}$ SPL.

Comparing the values of intervals I-III, III-V and I-V recorded after the stimulation of the left ear with those recorded after the stimulation of the right ear for the same intensity of the sound (Table 2), we did not notice significant differences $(P>0.05)$. The comparison of the values of intervals recorded after binaural and left monaural stimulation showed significant differences for intervals I-III and I-V only at sound levels of 70, 80 and 90 $\mathrm{dB}$ SPL. Comparing the values of the intervals recorded after binaural and right monaural stimulation significant differences were found for intervals I-III and I-V at $90 \mathrm{~dB}$ SPL as well as I-V and III-V at $80 \mathrm{~dB}$ SPL. 


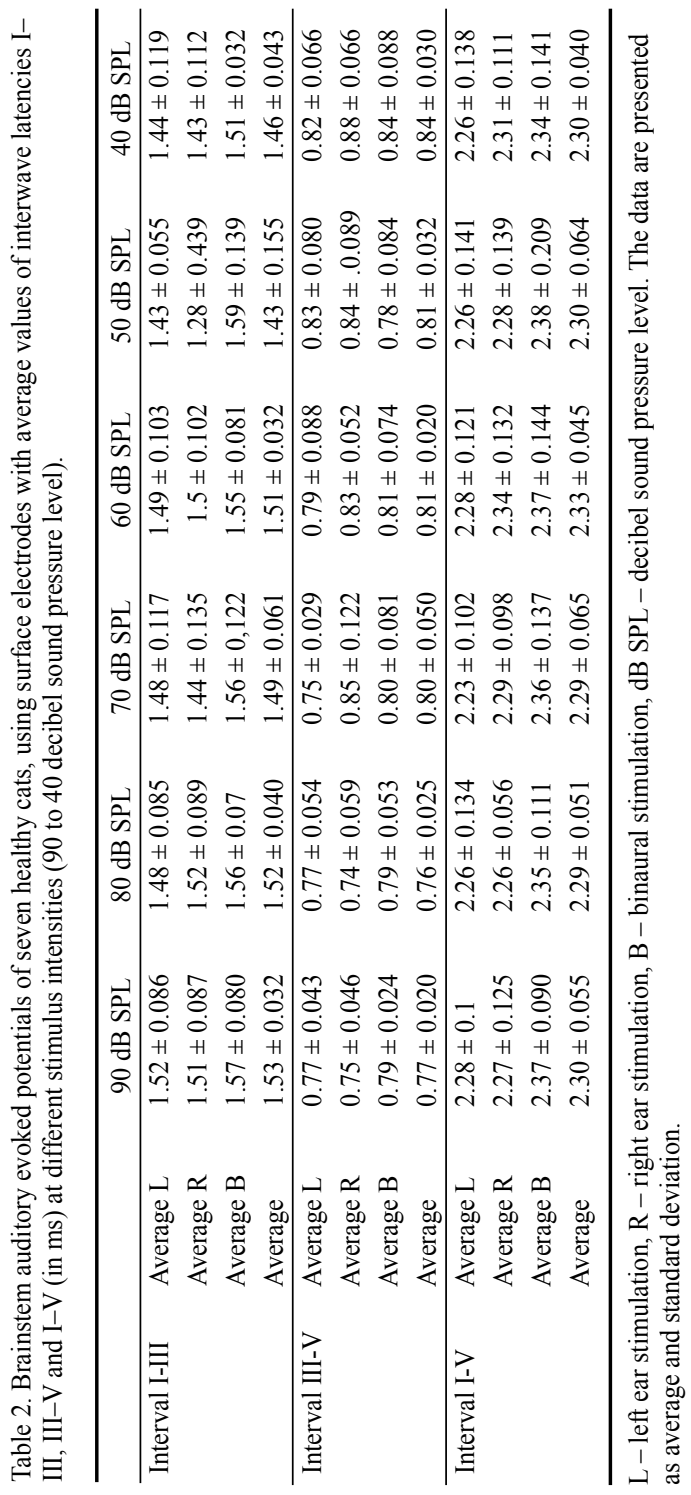

\section{Discussion}

This study served to evaluate the technique of recording BAER with surface electrodes, and its potential in clinical use by analysis of data obtained from normal cats under various aural stimulation conditions.

Our recorded latencies are lower than those described previously (Cauzinille 1997), possibly as a consequence of direct influence of using surface electrodes (Jost et al. 1994). As expected, the wave's latency decreased inversely with the stimulus intensity. The observed exception for intensities higher than $80 \mathrm{~dB}$ SPL may suggest the existence of an intensity-dependent neural summation in the structures involved in the transmission of the auditory stimulus with a maximal response at $80 \mathrm{~dB}$ SPL (minimal latency).

The differences observed between latencies III and V at binaural and monoaural stimulation (at least for intensities higher than $70 \mathrm{~dB}$ SPL) reflect the delay in the transmission of the impulse on the ascending pathways (Ungan et al. 1997; Kent et al. 2010). Since wave III originates in the ventral and dorsal nuclei of the trapezoid body, paired structures with several connections, this confirms that the auditory stimulus is firstly processed at this level. The delay seen for wave $\mathrm{V}$ latency reflects a secondary analysis of the information at the level of the two caudal colliculi (DeLahunta 1983). Caird and Klinke (1987) showed that a lesion located in one caudal colliculus in cats will cause a delay or absence of wave $\mathrm{V}$, after the stimulation of the contralateral ear. Batra and Fitzpatrick (2002) showed on rabbits a filter effect of the ventral nucleus of the lateral lemniscus. Therefore, there is a possibility that caudal colliculi have no inhibitory effect able to increase the latency of wave $\mathrm{V}$ after binaural stimulation, compared to monaural stimulation. The prolongation of wave $\mathrm{V}$ latency could be the result of active involvement of the nuclei of the lateral lemniscus alone. Regarding the morpho-physiological substrate of wave IV (formerly located in the lateral lemniscal pathways) in dogs, Wilson and Mills (2005) stated that it must be considered in conjunction with wave V (the wave IV-V complex) because of the small size of nuclei and the reduced likelihood of simultaneous 
neuronal firing, in light of the innervation from many different pathways. We conclude that although an analysis of the auditory stimuli could take place in the caudal colliculi, the changes of the latency of wave $\mathrm{V}$ may be the result of active involvement of the nuclei of the lateral lemniscus.

Despite the limited number of animals, this study has shown that the BAER using surface electrodes can be an accurate and repeatable method to assess the integrity of the pathways involved in the transmission of acoustic information through the brainstem in cats. Also, the results reflect that in the case of binaural stimulation there is a joint analysis of auditory information in the nuclear structures involved in the genesis of waves III and V, respectively. The unchanged latencies observed for wave III and V in the case of stimulation with stimulus higher than $80 \mathrm{~dB}$ SPL may be an important aspect in clinical evaluation were small changes in latencies values may be an objective factor in health status evaluation.

\section{Acknowledgements}

Researches financed from the grant PN II 62-085/2008, by Romanian National Center for Program Management (UEFISCDI), and grant PD 628/2010 (UEFISCDI).

\section{References}

Arnold S 2007: The auditory brainstem response. In: R. Roeser, H. Hosford-Dunn, \& M. Valente's (Eds.) Auditory: Diagnosis, treatment strategies, and practice management (2nd ed.). New York: Thieme-Medical Publishers.

Batra R, Fitzpatrick DC 2002: Monaural and binaural processing in the ventral nucleus of the lateral lemniscus: a major source of inhibition to the inferior colliculus. Hear Res 168: 90-97

Besalti O, Sirin YS, Pekcan Z 2008: The effect of chronic otitis externa-media on brainstem auditory evoked potentials in dogs. Acta Vet Brno 77: 615-624

Caird DM, Klinke R 1987: The effect of inferior colliculus lesions on auditory evoked potentials. Electroencephalogr Clin Neurophysiol 68: 237-240

Cauzinille L 1997: Measure of brainstem auditory evoked response: an objective method to test audition (in French). Point Vet 28: 37-40

Cvejic D, Steinberg TA, Kent MS, Fischer A 2009: Unilateral and bilateral congenital sensorineural deafness in client-owned pure-breed white cats. J Vet Intern Med 23: 392-395

De Lahunta A 1983: Auditory system - Special somatic afferent system. In: De Lahunta A: Veterinary neuroanatomy and clinical neurology, $2^{\text {nd }}$ ed. W.B. Saunder Co., St. Louis, pp. 304-307

Delack JB 1984: Hereditary deafness in the white cat. Compend Contin Educ Pract Vet 6: 609-619

Geigy CA, Heid S, Steffen F, Danielson K, Jaggy A, Gaillard C 2007: Does a pleiotropic gene explain deafness and blue irises in white cats? Vet J 173: 548-553

Jost WH, Ecker KW, Schimrigk K 1994: Surface versus needle electrodes in determination of motor conduction time to the external anal sphincter. Int J Colorectal Dis 9: 197-199

Kent M, Platt SR, Schatzberg SJ 2010: The neurology of balance: Function and dysfunction of the vestibular system in dogs and cats. Vet J 185: 247-58

Poma R, Chambers H, da Costa RC, Konyer NB, Nykamp S, Dobson H, Milgram NW 2008: MRI measurement of the canine auditory pathways and relationship with brainstem auditory evoked responses. Vet Comp Orthop Traumatol 21: 238-242

Strain GM 2007: Deafness in blue-eyed white cats: the uphill road to solving polygenic disorders. Vet J 173: 471-472

Ungan P, Yagcioglu S, Ozmen B 1997: Interaural delay-dependent changes in the binaural differences potential in cat auditory brainstem response: implications about the origin of the binaural interaction. Hear Res 106: 66-82

Wilson JW, Mills PC 2005: Brainstem auditory-evoked response in dogs. Am J Vet Res 66: 2177-2187 